\title{
On the possibility of partial matter mixing in rotating massive stars.
}

\author{
Eugenij I. Staritsin \\ Astronomical observatory, Ural State University, \\ pr. Lenina 51, Yekaterinburg, 620083 Russia
}

\begin{abstract}
The principal role of strong horizontal diffusion in overcoming the inhibiting effect of mean molecular weight gradient and in producing partial matter mixing between convective core and radiative envelope of a rotating star, is shown.
\end{abstract}

The effect of strong horizontal turbulent diffusion on the vertical transport of hydrogen (and helium) during the main sequence evolution of a rotating $16 \mathrm{M}_{\odot}$ star is studied. The horizontal shear created by meridional circulation in a rotating star produces anisotropic turbulence with strong horizontal diffusivity (Maeder 1997; Talon \& Zahn 1997). To look for the effect of this horizontal diffusion in a qualitative way we take the horizontal component of turbulent diffusivity as a parameter. The vertical component of turbulent viscosity $\nu_{v}$ is defined for several cases of initial state of rotation and for different values of horizontal component of turbulent diffusivity, on the base of the theory developed by Talon \& Zahn (1997). The distribution of the angular velocity of rotation in the initially chemically homogeneous main-sequence model was specified in the form

$$
\Omega= \begin{cases}\Omega_{c}, & \text { if } M_{r} \leq M_{c} ; \\ \Omega_{c}-b\left(M_{r}-M_{c}\right), & \text { if } M_{r}>M_{c},\end{cases}
$$

where $\Omega_{c}$ and $M_{c}$ are the angular velocity of rotation and the mass of the convective core, $M_{r}$ is the mass inside of isobar of averaged radius $r$. We calculated the evolution of a $16 \mathrm{M}_{\odot}$ star on the main sequence for $\alpha^{0}=1$ and 2 in the initial model, where $\alpha^{0}=\Omega_{c} / \Omega_{s}$ (Staritsin 2001). In both cases, the angular velocity of the surface rotation in the initial model was $\Omega_{s}=0.57210^{-4} \mathrm{~s}^{-1}$, which corresponds to a linear rotational velocity at the equator of $\sim 200 \mathrm{~km} \mathrm{~s}^{-1}$. Two values, $10^{10}$ and $10^{12} \mathrm{~cm}^{2} \mathrm{~s}^{-1}$, are taken for the horizontal component of turbulent diffusivity $D_{h}$.

Turbulence occurs in the outer radiative zone, including the layers where mean molecular weight varies with depth. Turbulence transports hydrogen from the radiative zone to the convective core. The mixing intensity in the turbulent envelope depends on both the initial rotation of star and the value of horizontal component of turbulent diffusivity. The more matter is mixed in the radiative zone, the more additional hydrogen is burned in the convective core. The products of hydrogen burning are carried out of the convective core by turbulence and spread in the radiative zone. The energy flux increases due to the increase of the helium content. As a result the decrease of the convective core mass is less 

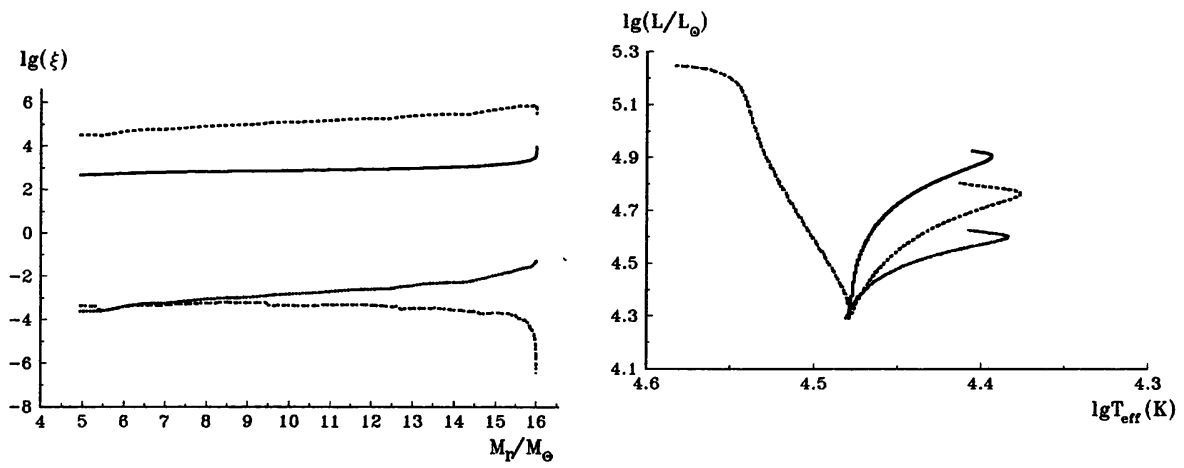

Figure 1. Left: physical conditions in the radiative envelope of a $16 \mathrm{M}_{\odot}$ model with central hydrogen content $X_{c} \simeq 0.1$ computed for the case $\left(\alpha^{0}, D_{h}\right)=\left(2,10^{10}\right)$ : the molecular viscosity: continuous line; the Reynolds number: dashed line; the parameter $\Gamma$ : dot-dash line; the ratio of $\nu_{v}$ to $D_{h}$ : dotted line. Right: evolutionary tracks for $16 \mathrm{M}_{\odot}$ models for the following cases: no rotation: dotted line; $\left(\alpha^{0}, D_{h}\right)=\left(1,10^{10}\right)$ : dashed line; $\left(\alpha^{0}, D_{h}\right)=\left(2,10^{10}\right)$ : continuous line; $\left(\alpha^{0}, D_{h}\right)=\left(1,10^{12}\right)$ : dot-dash line.

than in the case of non-rotating star. This effect is ascribed to 'overshooting' when one calculates the non-rotating stellar evolution. The helium content by mass in the stellar surface increases by $0.006-0.306$ to the end of main sequence evolution, depending on the mixing intensity in the radiative zone.

The physical conditions in turbulent radiative zones are shown in Figure 1a for the model with central hydrogen content $X_{c} \simeq 0.1$ in the case of $\left(\alpha^{0}, D_{h}\right)=\left(2,10^{10}\right)$. The molecular viscosity in turbulent radiative zones is evaluated using expression for hydrogen plasma (Lang 1974). . The Reynolds number in radiative zones exceeds 40000 ; thus the molecular viscosity is not sufficient to suppress the turbulence in the radiative zone. The parameter $\Gamma$ of mixing-length theory is very small, meaning that turbulence does not contribute to transport of energy. The ratio of $\nu_{v}$ to $D_{h}$ is small as it should be in case of anisotropic turbulence.

The evolutionary tracks for $16 \mathrm{M}_{\odot}$ models and sets of $\left(\alpha^{0}, D_{h}\right)$ are shown in Figure 1b. Partial matter mixing between convective core and radiative zones enhances the amplitude of the luminosity increase during hydrogen burning; the luminosity increase grows monotonously with the mixing intensity. The evolutionary increase of radius firstly grows with the mixing intensity. But for the case of the highest intensity of partial matter mixing, when $\left(\alpha^{0}, D_{h}\right)=$ $\left(1,10^{12}\right)$, the evolutionary increase of radius is even less than in the case of evolution without rotation.

\section{References}

Lang, K.R. 1974, Astrophysical Formulae, Vol. 1 (Berlin: Springer), ch.3

Maeder, A. 1997, A\&A 321, 134

Staritsin, E.I. 2001, Astron. Reports 45, 467

Talon, S., Zahn, J.-P. 1997, A\&A 317, 749 